ARTICLE

https://doi.org/10.1038/s41467-019-10637-8

\title{
Long-read sequencing unveils IGH-DUX4 translocation into the silenced IGH allele in B-cell acute lymphoblastic leukemia
}

Liqing Tian (1) ${ }^{1}$, Ying Shao ${ }^{1}$, Stephanie Nance ${ }^{2}$, Jinjun Dang ${ }^{2}$, Beisi Xu (i) ${ }^{1}$, Xiaotu Ma ${ }^{1}$, Yongjin Li ${ }^{1}$, Bensheng Ju', Li Dong${ }^{1}$, Scott Newman (1) ${ }^{1}$, Xin Zhou ${ }^{1}$, Patrick Schreiner ${ }^{1}$, Elizabeth Tseng ${ }^{3}$, Ting Hon ${ }^{3}$, Meredith Ashby ${ }^{3}$, Chunliang $\mathrm{Li}^{4}$, John Easton ${ }^{1}$, Tanja A. Gruber ${ }^{2}$ \& Jinghui Zhang ${ }^{1}{ }^{1}$

IGH@ proto-oncogene translocation is a common oncogenic event in lymphoid lineage cancers such as B-ALL, lymphoma and multiple myeloma. Here, to investigate the interplay between IGH@ proto-oncogene translocation and IGH allelic exclusion, we perform long-read whole-genome and transcriptome sequencing along with epigenetic and 3D genome profiling of Nalm6, an IGH-DUX4 positive B-ALL cell line. We detect significant allelic imbalance on the wild-type over the IGH-DUX4 haplotype in expression and epigenetic data, showing $I G H$ DUX4 translocation occurs on the silenced IGH allele. In vitro, this reduces the oncogenic stress of DUX4 high-level expression. Moreover, patient samples of IGH-DUX4 B-ALL have similar expression profile and IGH breakpoints as Nalm6, suggesting a common mechanism to allow optimal dosage of non-toxic DUX4 expression.

\footnotetext{
${ }^{1}$ Department of Computational Biology, St. Jude Children's Research Hospital, Memphis, TN 38105, USA. ${ }^{2}$ Department of Oncology, St. Jude Children's Research Hospital, Memphis, TN 38105, USA. ${ }^{3}$ Pacific Biosciences, Menlo Park, CA 94025, USA. ${ }^{4}$ Department of Tumor Cell Biology, St. Jude Children's Research Hospital, Memphis, TN 38105, USA. Correspondence and requests for materials should be addressed to J.Z. (email: jinghui.zhang@stjude.org)
} 
n B-cell development, the allelic exclusion of IGH (immunoglobulin heavy chain) follows a one B-cell, one antibody paradigm by first epigenetically silencing both alleles, then activating one allele during VDJ recombination so that functional $I G H$ is expressed from only one active $I G H$ allele in pre-B (precursor B) cells ${ }^{1-4}$. In lymphoid lineage cancer like B-cell acute lymphoblastic leukemia (B-ALL) ${ }^{5}$, lymphoma ${ }^{6}$, and multiple myeloma ${ }^{7}, I G H @$ proto-oncogene translocation is a common driver event. There is, however, limited knowledge about the interplay between IGH@ oncogenic translocation and allelic exclusion of $I G H$ (i.e., one allele is active, and the other is silenced) partly due to the technical challenges in delineating allelic specificity at the highly complex IGH locus (standard nextgeneration sequencing [NGS] technology can only generate shortread sequence data ranging from 100 to $150 \mathrm{bp}$ ).

$I G H-D U X 4$ translocation is one such oncogenic event, defining a B-ALL subtype with a distinct expression profile ${ }^{8-10}$. DUX4 is a transcription factor located within the GC-rich D4Z4 repeat array at the subtelomeric regions of $4 \mathrm{q} 35$ and $10 \mathrm{q} 26$, which are characterized by high levels of repression ${ }^{11,12}$. It is expressed only during events associated with major chromatin relaxation, i.e., in early embryos at cleavage stage (2/4/8-cell embryos) ${ }^{13}$ or after loss of repression of the D4Z4 macrosatellite repeat in myoblasts of individuals with facioscapulohumeral muscular dystrophy (FSHD) ${ }^{14,15}$. The IGH-DUX4 subtype accounts for $\sim 7 \%$ of pediatric B-ALLs in which an inter-chromosomal translocation repositions DUX4 within the vicinity of the $I G H$ enhancer $(\mathrm{E} \mu)$, the native enhancer of $I G H$ gene $(\operatorname{Ig} \mu)$ in pre-B cell ${ }^{8,9,16}$. IGHDUX4 translocation is a clonal event acquired early in leukemogenesis of this B-ALL subtype, resulting in an aberrant activation of DUX4 which is absent from other B-ALL subtypes ${ }^{8,9}$.

In this study, we investigate the interplay between IGH@ oncogenic translocation and allelic exclusion of $I G H$ in Nalm6, a B-ALL cell line that harbors IGH-DUX4 translocation. We use long-read technology which overcomes the limitation of shortread sequencing to evaluate the allele specificity of gene expression. Epigenetic states and enhancer-promoter interactions are analyzed using data generated from ChIP-seq, ATAC-seq, and 3$\mathrm{D}$ genome assays. Significant allelic imbalance is detected on the wild type over the IGH-DUX4 haplotype, showing that IGH$D U X 4$ translocation occurs on the silenced $I G H$ allele. In vitro assays suggest this may reduce the oncogenic stress of high-level expression of DUX4. Patient samples of IGH-DUX4 B-ALL have similar expression profiles and IGH breakpoints as Nalm6, suggesting this could be a common mechanism; further analyses of haplotype structure and epigenetic profiling are required.

\section{Results}

Expression of DUX4 and Ig $\mu$ in IGH-DUX4 B-ALLs. Using published RNA-seq data generated from 32 B-ALL patient samples that harboring IGH-DUX4 translocation and human embryos $^{9,13,17}$, we found that DUX4 expression is much higher compared with normal expression in human cleavage stage embryos (the median FPKM [fragments per kilobase of transcript, per million mapped reads] 145.4 vs. 6.65 , Fig. 1a). Typically this would suggest that the translocation would be into the active $I G H$ allele. Intriguingly, however, DUX4 expression was also much lower than Ig $\mu$ in these B-ALL samples (the median FPKM 145.4 vs. 661.9, Fig. 1b) - raising an alternative possibility that IGH-DUX4 translocation might have instead occurred on the silenced $I G H$ allele.

Selection of Nalm6 for genomic and epigenomic profiling. Nalm6, an IGH-DUX4 B-ALL cell line, was established from a 19-year-old patient with a near diploid B-ALL genome (http://bioinformatics.hsanmartino.it/cldb/cl3632.html). Consistent with our observation in IGH-DUX4 B-ALL patient samples, DUX4 expression in Nalm6 is much higher compared with its normal expression in human cleavage stage embryos (FPKM 41.3 vs. 6.65, Fig. 1a) and much lower than Ig $\mu$ (FPKM 41.3 vs. 638.2, Fig. 1b), making the cell line a goodmodel for evaluating allele specificity of the IGH-DUX4 translocation.

To resolve the mapping ambiguity caused by high repeat content in IGH and DUX4 regions, we profiled Nalm6 using three different approaches: (1) Iso-Seq, a method that generates fulllength transcripts; (2) 10X Chromium whole-genome sequencing (WGS), which incorporates barcodes into large DNA fragments prior to WGS thereby producing the so-called "digital" long WGS reads; and (3) stranded total RNA-seq. We also performed epigenetic and chromosome conformation capture assays to evaluate the allele specificity of epigenetic states and enhancer-promoter interactions. These include (1) H3K27ac ChIP-seq, ATAC-seq, and whole-genome bisulfite sequencing (WGBS); (2) genome-wide 3-D genome assays including $\mathrm{Hi}-\mathrm{C}$ and $\mathrm{H} 3 \mathrm{~K} 27 \mathrm{ac} \mathrm{HiChIP}$; and (3) deep sequencing of targeted chromosome conformation capture (Capture-C).

Allele specificity of $\mathbf{I G H}-\mathbf{D U X} 4$ translocation. A previous report has shown that two DUX4 copies were translocated between IGHJ4 and IGHD2-15 in Nalm6, resulting in expression of a DUX4 protein with its last 16 amino acids replaced by $I G H^{8}$. Incorporating this knowledge in our analysis, we first assembled the haplotype of IGH-DUX4 translocation by selecting reads near the rearranged region using Chromium molecular barcodes. This resulted in a $\sim 23 \mathrm{~kb}$ DNA sequence comprised of $I G H M$, the $I G H$ intronic enhancer $(\mathrm{E} \mu)$, two copies of the translocated DUX4 sequence, and flanking sequences near the two translocation breakpoints (Supplementary Fig. 1a). The translocated DUX4 sequence shares $100 \%$ sequence identity to DUX4L13 located on chromosome 10 while its best match on chromosome 4 is the DUX4L4 locus with $99.67 \%$ (four mismatches) sequence identity. RNA-seq reads share $100 \%$ identity to the assembled DUX4 contig, supporting exclusive transcription of DUX4L13 activated by translocation from chromosome 10 to $I G H$ in Nalm6 (Supplementary Fig. 2). The $I G H$ DUX4 translocation in Nalm6 therefore occurred between chromosomes 10 and 14 .

By mapping long Iso-Seq RNA reads to this assembled $I G H$ DUX4 haplotype, we identified full-length transcripts representing functional Ig $\mu$ IGH-DUX4 fusion, and antisense DUX4 transcription, and all Iso-Seq RNA reads that contain DUX4 are chimeric and can be mapped to the assembled IGH-DUX4 haplotype (Supplementary Fig. 1b). The antisense DUX4 transcript is comprised of an antisense DUX4 segment joined to the $I G H$ constant region (IGHM), which is also contained in the functional Ig $\mu$ transcript. Bidirectional transcription of sense and antisense DUX4 was also confirmed in our stranded RNA-seq data (Supplementary Fig. 2).

The IGHM locus is diploid in Nalm6 and contain three heterozygous germline SNPs (rs1059713, rs1136534, and rs3751511). Haplotype phasing from 10X Chromium WGS data demonstrate two haplotypes harboring alleles "T-G-G" and "AA-A" of these three SNPs. The full-length transcripts generated by Iso-Seq showed that functional Ig $\mu$ was transcribed exclusively from the "A-A-A" haplotype (i.e., the wild type without rearrangement) while the antisense $D U X 4$ was transcribed from the "T-G-G" haplotype that harbors the IGH-DUX4 translocation (Fig. 2a). Therefore, IGH-DUX4 translocation and functional Ig $\mu$ are on different $I G H$ alleles. 
a

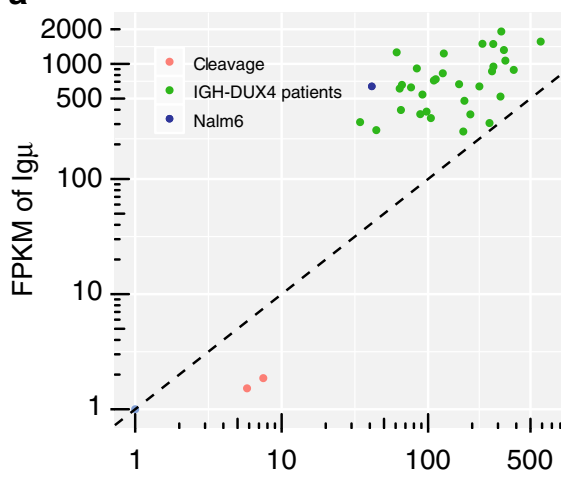

b 2000 .

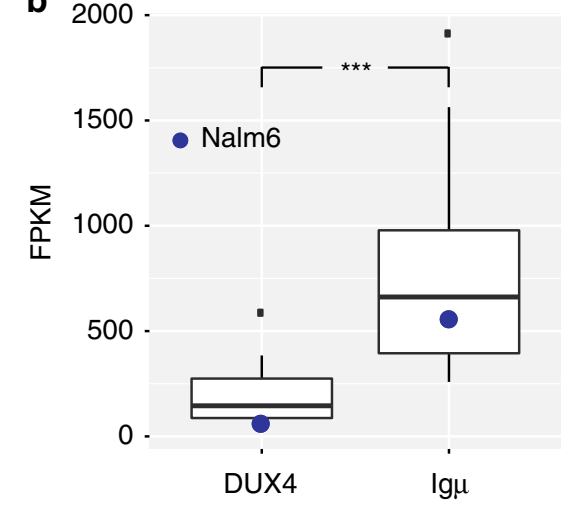

FPKM of DUX4

Fig. 1 Expression of the translocated DUX4 and Ig $\mu$ in IGH-DUX4 B-ALLs. a DUX4 and Ig $\mu$ expression in human embryonic cleavage cells, 32 IGH-DUX4 BALL patients, and Nalm6 cell line are shown in red, green and blue, respectively. b Box plot of DUX4 and Ig $\mu$ expression level in the 32 IGH-DUX4 B-ALL patients and Nalm6 cell line (blue dots). Median FPKM of DUX4 and Ig $\mu$ in 32 patients are 145.4 and 661.9, respectively. One-tailed, paired sample $t$-test was performed: ${ }^{\star \star \star} p<0.001$. Boxes show the first to third quartile with median, whiskers encompass 1.5 times of the interquartile range, and data beyond that threshold indicated as outliers
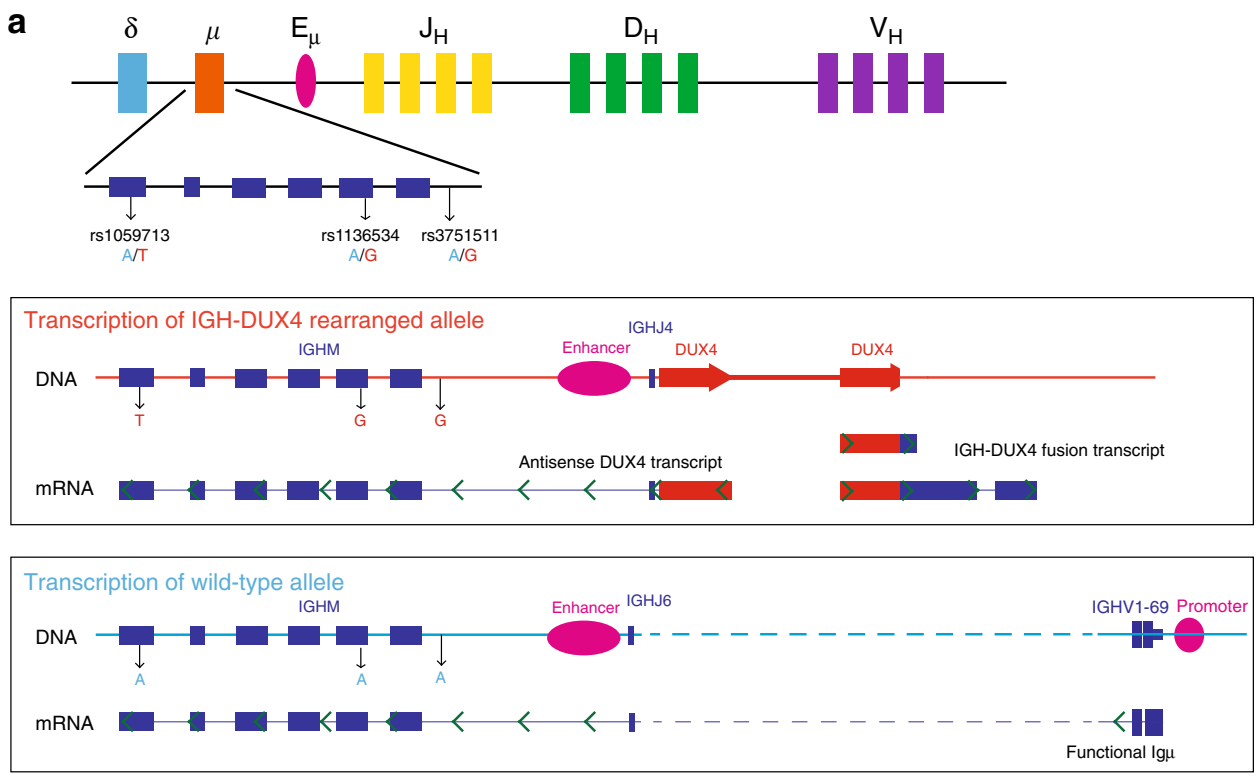

b

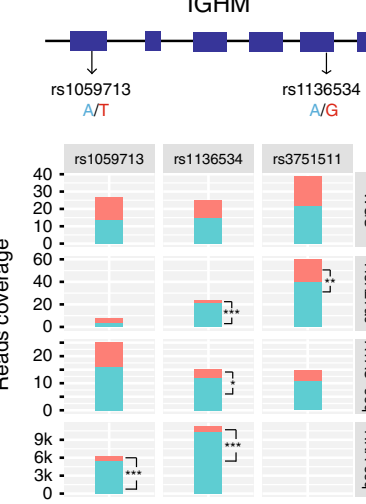

C

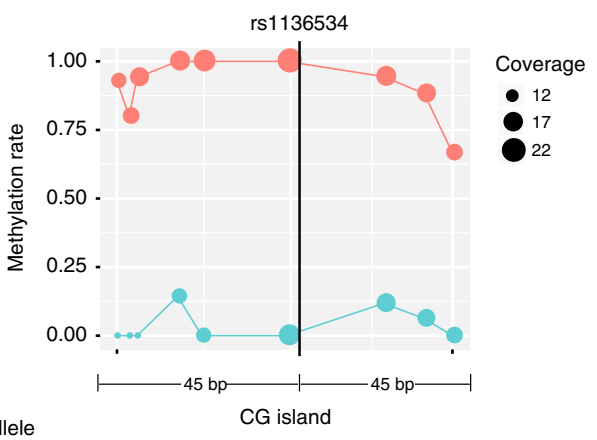

Fig. 2 Allele specificity of IGH-DUX4 translocation and functional Ig $\mu$. a Diagram of DUX4 and IGH gene expression at two alleles in Nalm6. Functional Ig $\mu$ and antisense DUX4 are expressed from different alleles. Green arrows in transcripts indicate the direction of transcription. $\mathbf{b}$ Allelic imbalance of H3K27ac ChIP-seq, ATAC-seq, and RNA-seq data in the three heterozygous SNPs (rs1059713, rs1136534, rs3751511) located at IGHM. For RNA-seq, read-out is available only for the two exonic SNPs (rs1059713, rs1136534). One-tailed binomial test (expected probability 0.5 ) was performed: ${ }^{*} p<0.05,{ }^{\star \star} p<0.01$, ${ }^{\star \star \star} p<0.001$. $\mathbf{c}$ Evaluation of allelic imbalance of DNA methylation near the SNP rs1136534 located in a CG island region. Each CpG site near the SNP is shown by a circle drawn in proportion to the reads coverage used for calculating the methylation ratio (see Methods) 
IGH-DUX4 translocation on silenced IGH allele. Prior studies have shown that functional Ig $\mu$ is expressed only from the active $I G H$ allele $^{1,3,18}$, suggesting that IGH-DUX4 translocation might be on the silenced IGH allele. Therefore, we examined allelic imbalance in RNA-seq, H3K27ac ChIP-seq, and ATAC-seq at the above-mentioned three heterozygous SNPs at the IGHM locus. Indeed, RNA-seq reads were significantly enriched on the wildtype IGH haplotype (i.e., the A-A-A haplotype) compared with the IGH-DUX4 haplotype (i.e., the T-G-G haplotype): expression of the wild-type allele is 7.4 and 10.4-fold of that of the IGHDUX4 haplotype allele at the two exonic SNPs rs1059713 and rs1136534, respectively. A similar pattern was found in H3K27ac and ATAC-seq reads which measure enhancer activity and chromatin accessibility, respectively (Fig. 2b). One SNP (rs1136534) was also located in a CG island region, and DNA methylation data shows hypomethylation of the wild-type haplotype in contrast to hypermethylation of the IGH-DUX4 haplotype (Fig. 2c). These data provided further support that the DUX4 translocation occurs on the silenced IGH allele in Nalm6.

Enhancer-promoter interaction at the two IGH haplotypes. Prior studies have shown that both Ig $\mu$ and the translocated DUX4 were regulated by the IGH enhancer $(\mathrm{E} \mu)^{8,16}$. We confirmed the interactions of $\mathrm{E} \mu$-Ig $\mu$ promoter and $\mathrm{E} \mu-D U X 4$ by performing genome-wide Hi-C (Fig. 3a) and H3K27ac HiChIP in Nalm6 (Supplementary Fig. 3). The Hi-C data demonstrate that the interaction of IGH locus on chromosome 14 with the DUX4 region on 10q26 is much broader than that with the DUX4 region on 4q35 (Supplementary Fig. 4), consistent with our finding that the translocated DUX4 was from chromosome 10 based on the assembled IGH-DUX4 haplotype and expressed transcripts by RNA-seq and Iso-Seq.

$\mathrm{E} \mu$-Ig $\mu$ promoter interaction measured by read-pair count in $\mathrm{Hi}-\mathrm{C}$ and H3K27ac HiChIP is 3- and 8-fold of that of the E $\mu$ DUX4 interaction, respectively (Fig. $3 c$, details in Methods section). The stronger $\mathrm{E} \mu$ and Ig $\mu$ interaction is not related to the distance-associated chromatin interaction decay ${ }^{19}$ because the distance between $\mathrm{E} \mu$ and $I g \mu$ promoter on the wild-type haplotype $(\sim 140 \mathrm{~kb})$ is in fact much longer than that $(\sim 4 \mathrm{~kb})$ between E $\mu$ and DUX4 on the IGH-DUX4 haplotype (Fig. 3b, see Methods). To verify this, we designed Capture-C with bait probes around $\mathrm{E} \mu$ and performed coverage-based peak analysis, which shows that $\mathrm{E} \mu$-Ig $\mu$ promoter interaction is 13 -fold higher than that of E $\mu$-DUX4 interaction (Fig. 3c, see Methods). The uniform pattern of weaker $\mathrm{E} \mu-D U X 4$ interaction emerging from $\mathrm{Hi}-\mathrm{C}$, HiChIP, and Capture-C data provides further support that $I G H-$ DUX4 haplotype was epigenetically silenced, consistent with allele-specific hypermethylation of this haplotype (Fig. 2c). The $\mathrm{E} \mu$-Ig $\mu$ promoter interaction was mono-allelic for the wild-type $I G H$ allele based on the read-out of $\mathrm{Hi}-\mathrm{C} / \mathrm{HiChIP} / \mathrm{Capture}-\mathrm{C}$ data at the three heterozygous SNPs located near Ig $\mu$ promoter (Supplementary Fig. 5b). This can be attributed to a reciprocal rearrangement between the 10q26 subtelomeric region and the IGH region unveiled by the Hi-C data (Supplementary Fig. 5, details in Methods section).

DUX4 overexpression leads to increased apoptosis. Our analysis on the transcriptome, epigenome and $3 \mathrm{D}$ genome shows that in Nalm6 the IGH-DUX4 translocation occurred on the silenced $I G H$ allele, which would result in reduced expression of DUX4 compared with the alternative, i.e., translocation to the active, wild-type allele. Overexpression of oncogenes in cells may cause replicative stress and activation of anti-oncogenic pathways leading to apoptosis, a process often referred to as "oncogenic stress"20,21. Previous studies have demonstrated that transduced expression of DUX4 in rhabdomyosarcoma cell lines led to apoptosis ${ }^{22}$. We therefore reasoned that the IGH-DUX4 translocation on the repressive haplotype would avoid high-level DUX4 expression if it were transcribed from the haplotype with an active $\mathrm{E} \mu$ which would not be tolerated in normal or cancer cells. To test this hypothesis, we introduced the DUX4 oncogene into hematopoietic cells by transduction of primary murine bone marrow cells with a retroviral vector that carries DUX4 and the eGFP reporter gene (Fig. 4a, b). Apoptosis, as measured by annexin V staining was determined in GFP high and low populations $36-48 \mathrm{~h}$ after transduction. We found a significant increase in apoptosis in cells with high levels of DUX4 compared with those with low levels as indicated by GFP levels $(p=0.0484)$. To further evaluate the effects of DUX4 overexpression in fully transformed leukemia cells, we introduced DUX4 into Nalm6 cells which already harbor the IGH-DUX4 translocation, confirming GFP-tagged DUX4 protein expression by western blot (Fig. 4c, d). The results showed that fully transformed leukemia cells would not tolerate further overexpression of DUX4 with a significant increase in apoptosis when comparing DUX4 transduced cells with the empty vector control $(p=0.0124)$. We therefore conclude that while low levels of the DUX4 protein are tolerable, high levels induce apoptosis providing a biologic rationale for expression from the repressive haplotype.

\section{Discussion}

By integrating DNA translocation breakpoints, epigenetic profiling, transcription and cell toxicity data in Nalm6, we propose the following model for IGH-DUX4 translocation during B-cell development (Fig. 5) based on current knowledge of stepwise epigenetic process that controls the allelic exclusion of $I G H^{1,3,18}$. B-cell development initiates from hematopoietic stem cells with both IGH alleles hypo-acetylated and silenced by DNA hypermethylation ${ }^{1,18}$. This is followed by the development of early progenitor B (pro-B) cells where both IGH alleles undergo D-to-J rearrangement. Structural variation (SV) breakpoints of $I G H-$ DUX4 in Nalm6 occurred in the IGH D-J junction, suggesting that the IGH translocation arise at the pro-B cell stage as demonstrated previously by SV breakpoint analysis in IGH rearranged multiple myeloma patient samples ${ }^{23}$. Consistent with this, prior studies by our group and the others showed that the IGH-DUX4 fusions are clonal events acquired early in leukemogenesis ${ }^{8,9}$. At this stage, both $I G H$ alleles remain methylated. In late pro- $\mathrm{B}$ and pre-B cell stage, one of the $I G H$ alleles is randomly selected for activation by demethylation and hyperacetylation followed by the VDJ rearrangement. If an IGH-DUX4 rearranged allele were selected for activation-which is possible as not all BALLs express functional $I G H^{24,25}$, the resulting highly expressed DUX4 would likely be too toxic to permit the survival of leukemia cells, leading to cell death (Fig. 4). By contrast, activation of the wild-type IGH allele would lead to moderate expression of DUX4 from the silenced IGH-DUX4 allele, ensuring leukemia cell viability.

Previously, Yasuda et al. has shown that knockdown of the IGH-DUX4 fusion would suppress proliferation of Nalm6 cells ${ }^{8}$, confirming the oncogenic potential of DUX4 expression in BALL. This, coupled with the oncogenic toxicity caused by overexpression of DUX4 presented in this study (Fig. 4), suggests that DUX4 expression in Nalm6 may follow the "Goldilocks principle"26_i.e., "just-right" levels are required as too-much will lead to apoptosis while too-little will lead to suppression of proliferation. Indeed, among patient samples that harbor IGH-DUX4 fusion, DUX4 expression represents a fraction of the Igu expression level $(26.8 \%+/-6.4 \% 95 \% \mathrm{CI})$ with a positive correlation (Pearson's $r=0.554, p$-value $=0.001$, two-tailed $p$ value by 


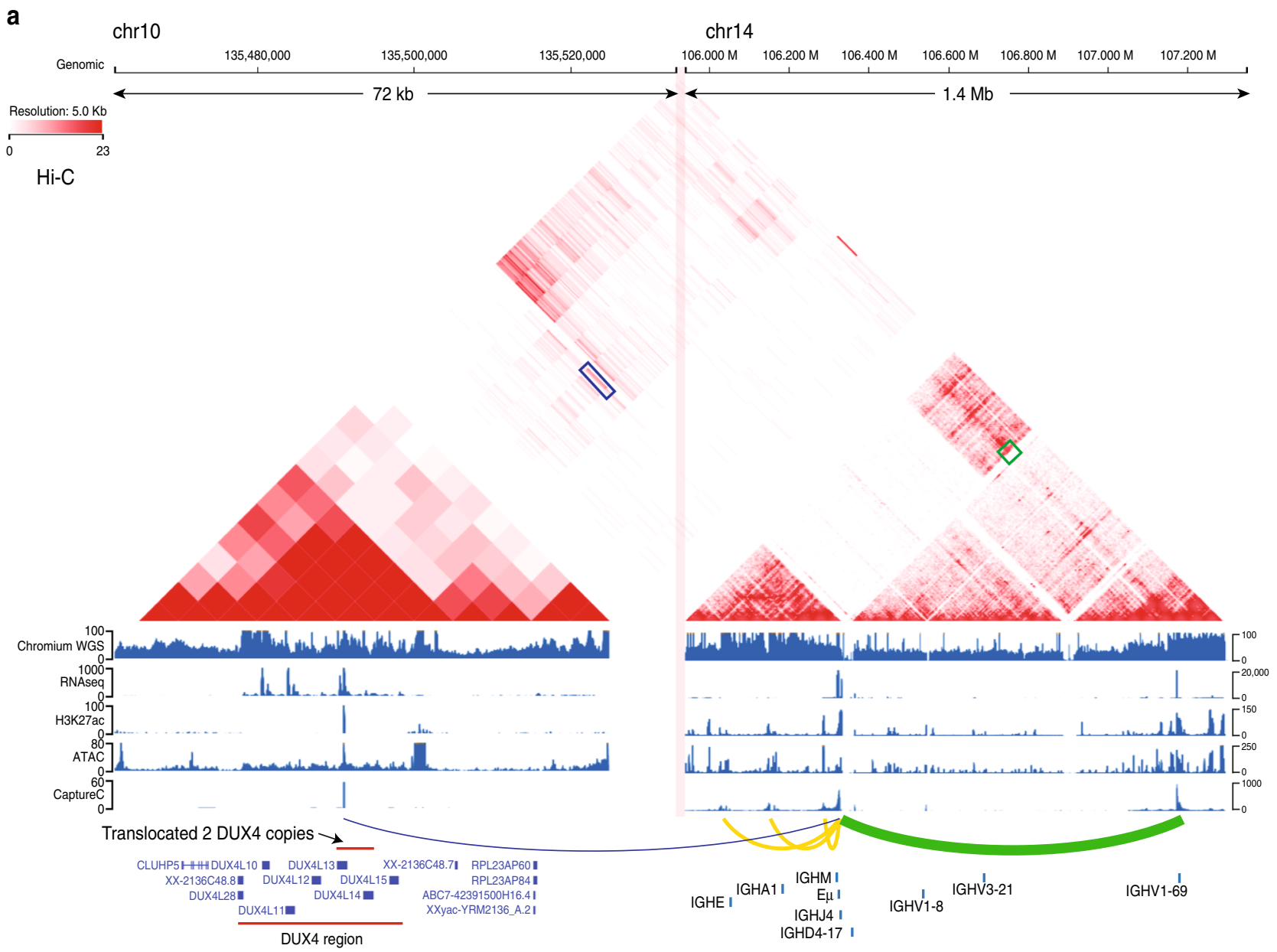

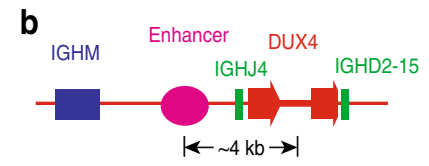

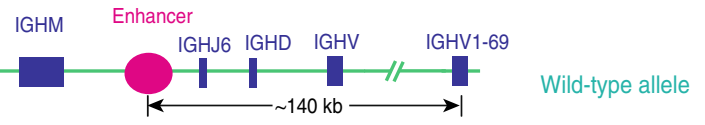

C
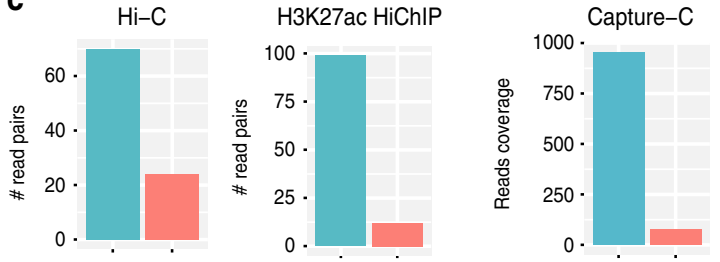

E $\mu$-DUX4 interaction

E $\mu$-Ig $\mu$ promoter interaction

Fig. 3 Allelic specificity of enhancer-promoter interaction in IGH-DUX4 haplotype versus wild-type haplotype in Nalm6. a Chromatin interaction plot from $\mathrm{Hi}-\mathrm{C}$ data at the DUX4 region $(72 \mathrm{~kb})$ on $10 \mathrm{q} 26$ and the $I G H$ region $\left(1.4 \mathrm{Mb}\right.$ ) plotted by the ProteinPaint program ${ }^{43}$. Different $y$-axis scale was used at DUX4 and IGH region for plotting RNA-seq, H3K27ac, ATAC-seq, and Capture-C. The E $\mu$-DUX4 interaction and E $\mu$-lg $\mu$ promoter interaction are marked as a blue and green box, respectively. In Capture-C data, the colored arcs represent regions interacting with $E \mu$, with thickness indicating interaction intensity. Yellow arcs show known $E \mu$-Ig $\mu$ interactions. The two translocated DUX4 copies were marked at DUX4L13 region, because this region is best matched to our assembled IGH-DUX4 haplotype. Details are in Methods section. b Diagrams of E $\mu$-DUX4 interaction and E $\mu$-Ig $\mu$ promoter interaction in different alleles. The genomic distances were estimated based on Chromium WGS data and the knowledge that the functional Ig $\mu$ is from the active IGH allele which undergoes VDJ recombination ${ }^{3}$. Details are in Methods section. c Comparison of supporting read pairs for Hi-C/H3K27ac HiChIP, the reads coverage for Capture-C between $\mathrm{E} \mu-D U X 4$ interaction and $\mathrm{E} \mu$-Ig $\mu$ promoter interaction. Multiple mapping issue at DUX4 regions was considered. The details are described in Methods section

the Pearson's correlation test) between Igu and DUX4 expression (Fig. 1a). DUX4 translocation to the silenced allele may thus provide the selective advantage required to achieve the precise level of expression to promote fitness of leukemia cells. In this regard, targeting of DUX4 by considering the potential for exploiting its oncogenic stress ${ }^{26,27}$ may provide a unique therapeutic angle for IGH-DUX4 B-ALL treatment.

Our analysis of RNA-seq data generated from 54 B-ALL pediatric patient samples with IGH@ translocation showed that
SV breakpoints on $I G H-D U X 4$ and $I G H$-CRLF2 translocation were highly enriched on IGH D-J junctions (red box on Supplementary Fig. 6) while expression of the target oncogenes, i.e., DUX4 (Fig. 1) and CRLF2 (Supplementary Fig. 7, data are from published paper ${ }^{17,28}$ ) is significantly lower than that of Ig $\mu$-both patterns match what we observed in Nalm6. The consistency between patient data and the Nalm6 cell line suggest that IGH@ proto-oncogene translocation on the silenced allele, discovered in Nalm6 through comprehensive analysis on haplotype structure 
a

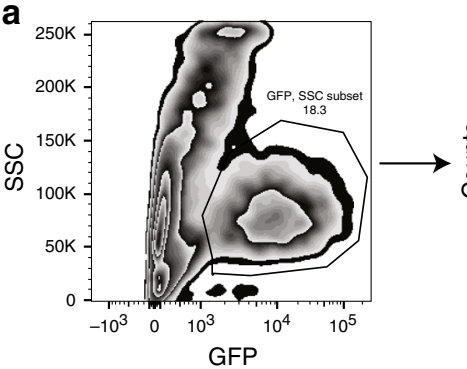

b

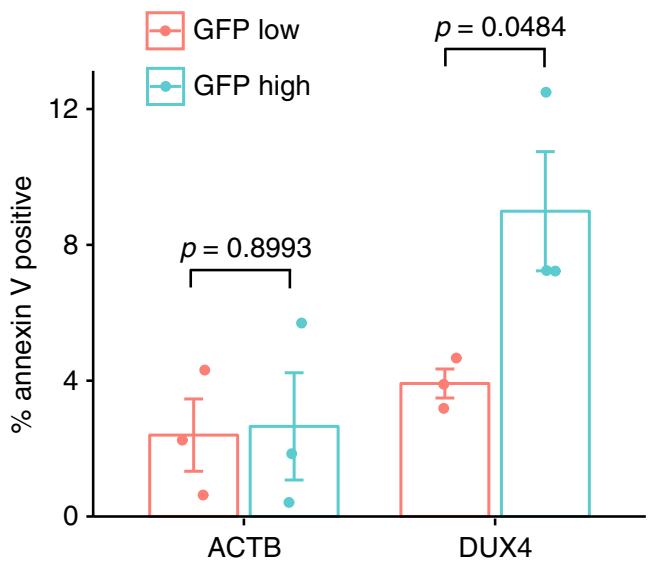

C

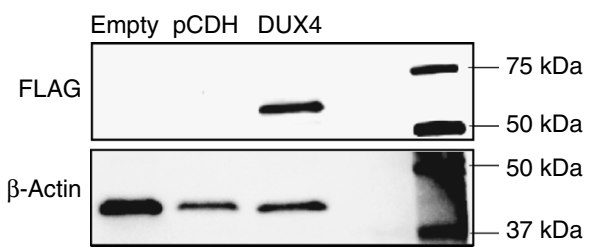

d

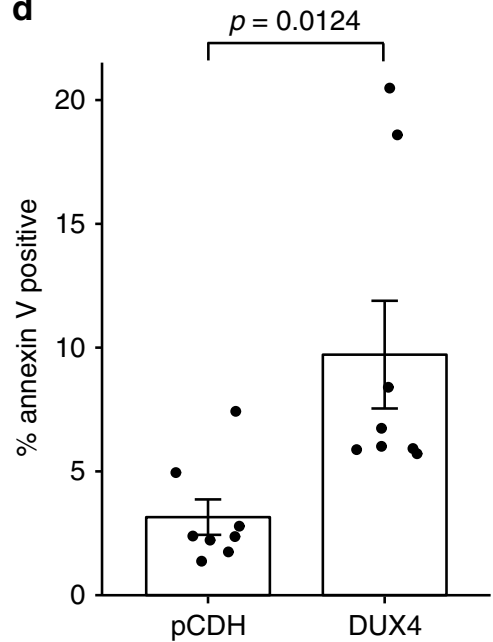

Fig. 4 DUX4 overexpression leads to an increased apoptosis. a Murine bone marrow cells were transduced with a retroviral vector containing DUX4 or beta actin (ACTB) as a control. Apoptosis was measured by Annexin V staining. Gating strategy is shown. GFP positive cells were gated on, the top $30 \%$ and bottom $30 \%$ mean fluorescence intensity (MFI) were selected and analyzed for annexin $\mathrm{V}$ staining. $\mathbf{b}$ Percent apoptotic cells as determined by annexin $\mathrm{V}$ staining in GFP low and GFP high murine bone marrow populations are shown. Data are combined from three independent experiments. c Nalm6 cells were transduced with a lentiviral vector containing GFP alone ( $\mathrm{pCDH}$ ) or FLAG tagged DUX4 IRES GFP (DUX4). Transduced cells were flow sorted for GFP positive populations followed by protein extraction and western blot analysis. Non-transduced cells were included as a control (Empty). Blots were stained using an anti-FLAG antibody (FLAG) and anti-beta actin ( $\beta$-actin). Sorted cells from three separate experiments were pooled for the western blot. d Apoptosis was measured by Annexin $V$ staining in flow sorted GFP positive cells. Data are combined from eight independent experiments. b, d Twotailed $p$ values calculated using the unpaired $t$-test are indicated. All data are represented as means with standard error of the mean

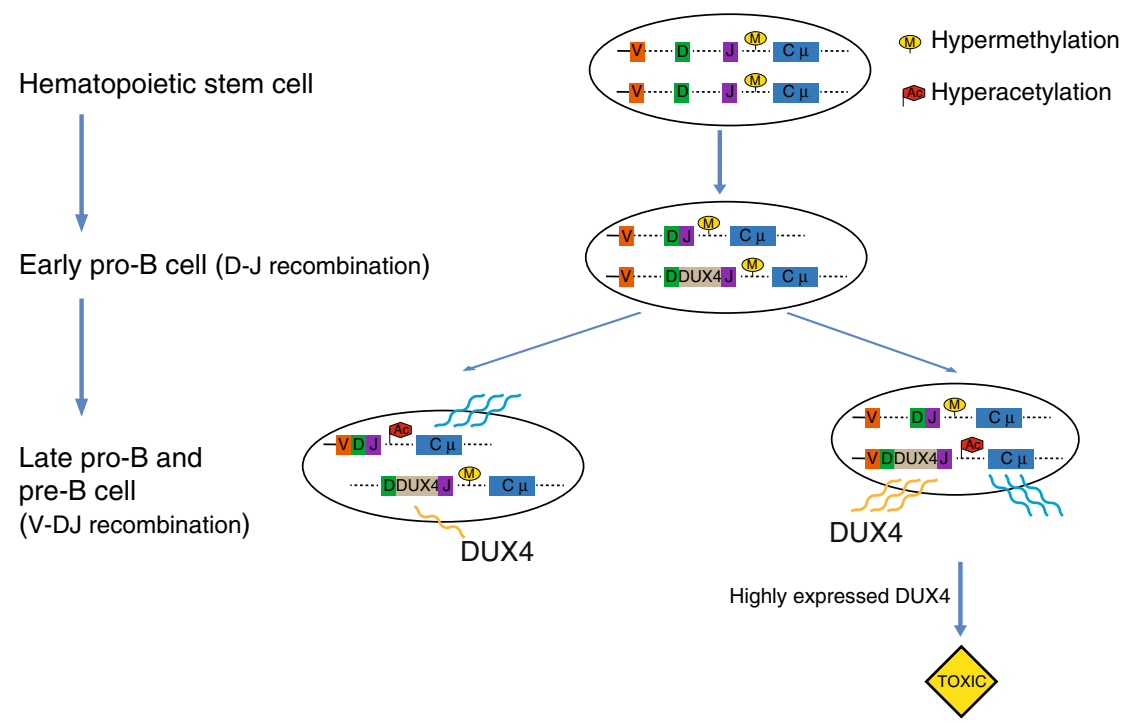

Fig. 5 A proposed model for the interplay between IGH-DUX4 translocation and IGH allelic exclusion during B-cell development 
and epigenetic profiling, could also occur in B-ALL patient samples. Further studies employing haplotype analysis and epigenomic profiling using patient samples or patient-derived xenograft mouse models are needed to provide additional support for this hypothesis.

\section{Methods}

Evaluation of gene expression level from RNA-seq. RNA-seq data (GSE85632) for human cleavage cells ${ }^{13}$ were downloaded from the GEO database by SRA toolkit v2.8.2-1. The data were aligned to hg19 (GRCh37-lite) using STAR v2.529. Published RNA-seq data of 32 IGH-DUX4 and 24 IGH-CRLF2 B-ALL patients were used for the analysis $9,17,28$, which were available in EGA (EGAS00001001923, EGAS00001000654) and dbGaP (phs000218). FPKM was calculated for each gene based on the gene models in GENCODE v19 using HTseqcount $^{30}$. Since there are many copies of DUX4 in the human genome, in order to evaluate DUX4 gene expression level, all the reads were mapped to DNA segment of a single DUX4 locus (DUX4L13 1267 bp length, chr10:135,490,575-135,491,841 in hg19), then the mapped read counts were used to calculate FPKM. Our local assembly of IGH-DUX4 haplotype derived from the Chromium WGS showed DUX4L13 on the reference genome is the best match to the translocated DUX4. For Ig $\mu$, different $B$ cells use different $V / D / J$ components; however, the constant region of $\operatorname{Ig} \mu(I G H M)$ is used in all pre-B cells ${ }^{1}$. The FPKM of IGHM was therefore used to evaluate Ig $\mu$ gene expression.

10x Genomics Chromium WGS in Nalm6 and haplotype phasing. High molecular weight genomic DNA of Nalm6 (ATCC, Manassas, VA, USA; the same origin below) was extracted using Qiagen MagAttract HMW DNA Kit. 1.0 ng HMW gDNA was input, and each molecule was captured by a gel bead which contained specific barcode to distinguish different molecules on 10X Genomics Chromium Controller, then amplified by isothermal incubation. The barcoded DNA was purified, then end repaired, dA-tailed and adaptor ligated using chromium genome library kit. Finally, the library was purified and enriched by index PCR amplification then sequenced paired-end 151 cycles on Illumina HiSeq4000.

The Long Ranger 2.0.0 pipeline (https://support.10xgenomics.com/genomeexome/software/pipelines/latest/using/wgs) was used to perform variant calling and phasing. First, we mapped the reads to hg19 with Lariat using default parameters. Second, Freebayes v0.9.21 was used to call haplotype-based variants. Finally, we filtered the variants using bcftools vl.1 (-e ' $(\% \mathrm{QUAL}<=15 \|(\mathrm{AF}[0]>0.5 \& \& \%$ $\mathrm{QUAL}<50))$ ' and -e ' $\left.(\mathrm{AO}[0]<2 \| \mathrm{AO}[0] /(\mathrm{AO}[0]+\mathrm{RO})<0.15)^{\prime}\right)$.

Local assembly of IGH-DUX4 translocation DNA. The Chromium molecular barcodes were used to select reads near the IGH-DUX4 rearranged region. First, the 10X Chromium WGS reads mapped to the $I G H$ region

(chr14:106,000,000-107,349,540 in hg19, from IGHA to the chromosome end) were selected. Second, we obtained the barcodes from the selected reads, only keeping barcodes with $\geq 20$ reads. Third, all the reads with these barcodes in the whole library were selected, even where the read was unmapped or was mapped to non-IGH regions. Fourth, de novo DNA assembly was performed based on the selected reads using Supernova v1.1.431 with parameter "output_pseudohap". Fifth, all the assembled contigs were mapped to GRCh38 using blat ${ }^{32}$. We used GRCh 38 for this analysis because the annotation on IGH and DUX4 is much improved on this more recent version of the reference genome. An $8063 \mathrm{bp}$ contig was mapped to the IGHM region (marked as a blue bar in Supplementary Fig. 1a). Yasuda et al. ${ }^{8}$ reported DNA sequences near the IGH-DUX4 breakpoints in Nalm6 (marked as green bar in Supplementary Fig. 1a) using PacBio sequencing. However, no contig was found to link IGHM and the known sequence at the rearranged region. A possible reason is that a $3.5 \mathrm{~kb}$ tandem repeat sequence existed in this linking region, making it difficult to fully assemble the entire region. Therefore, the reference genome sequence (chr14:105857149-105863252 in hg38) was used in this linking region (marked as a yellow bar in Supplementary Fig. 1a). We also found a 4637 bp contig span encoding DUX4 and the intergenic region between two DUX4 repeats. Based on this contig and the known sequences near breakpoints, the sequence of translocated DUX4 was manually constructed (marked as red bar in Supplementary Fig. 1a); the length ( $4670 \mathrm{bp}$ ) was consistent with that reported by Yasuda et al. ${ }^{8}$ (4663 bp). In summary, we manually assembled a 23,361 bp DNA segment, including IGHM, the IGH intronic enhancer $(\mathrm{E} \mu)$, the translocated DUX4 sequence, and the sequence near the breakpoints.

We mapped the $\sim 23 \mathrm{~kb}$ sequence to hg19 and found the assembled DUX4 region was best matched to DUX4L13. DUX4L13 showed exactly the same as our assembled sequence. The other DUX4 copies in hg19 showed at least one mismatch with the assembled sequence.

Iso-Seq and data analysis. One microgram of total RNA per reaction was reverse transcribed using the Clontech SMARTer cDNA synthesis kit. Four reverse transcription (RT) reactions were processed in parallel. PCR optimization was used to determine the optimal amplification cycle number for the downstream large-scale PCR reactions. A single primer (primer IIA from the Clontech SMARTer kit 5'-
AAGCAGTGGTATCAACGCAGAGTAC-3') was used for all PCR reactions postRT.

Large-scale PCR products were purified with either $1 \times$ or $0.4 \times$ AMPure $\mathrm{PB}$ beads ( $1 \times$ or $0.4 \times$ cDNA library hereafter) and the bioanalyzer was used for QC. Equimolar ratios of the $1 \times$ and $0.4 \times \mathrm{cDNA}$ libraries were pooled to generate the non-size-selected SMRTBell library. A total of $3.5 \mu \mathrm{g}$ of $1 \times \mathrm{cDNA}$ library was input into size fractionation using the Sage BluePippin system. A 4.5-10 kb size fraction was eluted, re-amplified and cleaned with AMPure PB beads. A SMRTBell library was constructed using $2.3 \mu \mathrm{g}$ of size-selected cDNA (https://www.pacb.com/wpcontent/uploads/Procedure-Checklist-Iso-Seq\%E2\%84\%A2-Template-

Preparation-for-Sequel-Systems.pdf).

The non-size-selected and size-selected Iso-Seq SMRTBell libraries were pooled in a 3:1 ratio and co-loaded on a single Sequel cell. A total of two SMRT cells (one $6 \mathrm{~h}$ and one $10 \mathrm{~h}$ movie) were sequenced on the PacBio Sequel platform using 2.0 chemistry.

After running Iso-Seq pipeline, 144,756 polished circular consensus sequences (CCS) reads (201-14,703 bp) were generated. Then all the reads were mapped to the assembled DNA segment using STAR v2. $5^{29}$ with recommended parameters for Iso-Seq (https://github.com/PacificBiosciences/IsoSeq_SA3nUP/wiki/OldTutorial:-Optimizing-STAR-aligner-for-Iso-Seq-data). The majority of the mapped reads contained IGHM exons (Supplementary Fig. 1b) and IGHV1-69 (not shown, because it is excluded in the assembled sequence), and these reads were in-frame coding for functional Ig $\mu$. Two IGH-DUX4 fusion transcripts were found, and the shorter sequence was supported by $3^{\prime}$ RACE experiment by Yasuda et al.$^{8}$. Two antisense DUX4 transcripts containing IGHM exons were also identified (Supplementary Fig. 1b).

RNA-seq/H3K27ac ChIP-seq/ATAC-seq in Nalm6 and data analysis. Total stranded RNA-seq in Nalm6 were from our previously published work ${ }^{9}$ and available in EGA (EGAS00001000654)

For ATAC sequencing, we followed the protocol established by Buenrostro et al. ${ }^{33}$. Briefly, around 50,000 Nalm6 cells were used for nuclei preparation. Chromatin fragmentation and library construction were carried out using the Nextera DNA sample preparation kit (Illumina, Cat\# FC-121-1030). DNA library sequencing was done on an Illumina Hiseq 2500 with 75 bp paired-end reads. The reads were trimming for Nextera adapter by cutadapt (version 1.9, paired-end mode, default parameter with " $-\mathrm{m} 6-\mathrm{O} 20$ ") and aligned to human genome hg19 (GRCh37-lite) by BWA (version 0.7.12-r1039, default parameter) ${ }^{34}$, duplicated reads were then marked with Picard (version 2.6.0-SNAPSHOT) ${ }^{35}$ and only nonduplicated proper paired reads have been kept by samtools (parameter "-q 1 -F 1804 " version 1.2) (36 $^{36}$ After adjustment of Tn5 shift (reads were offset were offset by +4 bp for the sense strand and -5 bp for the antisense strand) we separated reads into nucleosome free, mononucleosome, dinucleosome, tri-nucleosome as described in Buenrostro et al. ${ }^{37}$ by fragment size and generated bigwig files by using the center $80 \mathrm{bp}$ of fragments and scale to $30 \mathrm{M}$ nucleosome free reads. We observed reasonable nucleosome free peaks and pattern of mono-, di-, trinucleosome on IGV (version 2.4.13) ${ }^{38}$. The samples have more than $20 \mathrm{M}$ nucleosome free reads so we conclude the data qualities are good and have enough depth to not miss any chromatin accessible regions.

For the H3K27ac ChIP-seq, a frozen cell pellet containing 10 million cells was sent to Active Motif for ChIP and library preparation. The sample was divided into an aliquot for ChIP using an antibody to $\mathrm{H} 3 \mathrm{~K} 27 \mathrm{ac}$ (Active Motif) and an input control. Single-end sequencing was performed using an Illumina NextSeq 500 generating 76 cycles for each sequencing read. The reads were mapped to human genome ho19 (GRCh37-lite) by BWA (version 0.7.12-r1039, default parameter) ${ }^{34}$ duplicated reads were then marked with Picard (version 2.6.0-SNAPSHOT ${ }^{35}$ and only non-duplicated reads have been kept by samtools (parameter "-q 1 -F 1024" version 1.2) ${ }^{36}$. We followed ENCODE guideline ${ }^{39}$ for quality control. Briefly, we used SPP 40 to draw cross-correlation plot and calculated relative strand correlation value (RSC). We then estimate the fragment size from cross-correlation plot and extended each read to the estimated fragment size to generate bigwig files (normalized to 15 million unique mapped reads). Observed clear peak shape along with RSC $>1$ and $\sim 40 \mathrm{M}$ (ENCODE criterion $10 \mathrm{M}$ ) unique mapped reads, we conclude our IP sample for H3K27ac is good.

Whole-genome bisulfite sequencing in Nalm6 and data analysis. In total, 200 ng genomic DNA of Nalm6 was sheared around $400 \mathrm{bp}$ using Covaris LE220 and followed by bisulfite conversion using EZ DNA Methylation-Gold Kit. The fragmented, bisulfite-converted single-strand DNA was tailed and ligated of truncated adapter one to $3^{\prime}$ end, primer extended and truncated of adapter two at the bottom strand only. Finally, the library was purified and enriched by six cycles of index PCR amplification then sequenced paired-end 151 cycles on Illumina NovaSeq 6000

The reads were mapped to hg19 using bsmap v2.74 $4^{41}(-\mathrm{q} 20$-u -v 0.08 -w $100-\mathrm{z}$ 33). The mapping rate was $92.9 \%$, and the bisulfite conversion rate was $99.3 \%$ in mitochondria. For allele-specific DNA methylation analysis, in IGHM region, only the heterozygous SNPs rs1136534 was in CG island. All 9 CG positions near the SNP (left and right 45 bp region) were considered. Because the SNP rs1136534 located in CG content, only the reads with meaningful orientation overlapped with the SNP were considered. For each selected read, we first identified if the read was a 
wild type or IGH-DUX4 rearranged allele before assessing the number of methylated or unmethylated reads to define coverage and the methylation ratio at the 9 CG positions.

Hi-C and data analysis. The Nalm6 cell line was cultured under recommended conditions to about $80 \%$ confluence. Five million cells were crosslinked with $1 \%$ formaldehyde for $10 \mathrm{~min}$ at room temperature, then digested with 125 units of MboI, and labeled by biotinylated nucleotides and proximity-ligated. After reverse crosslinking, ligated DNA was purified and sheared to $300-500 \mathrm{bp}$, then ligation junctions were pulled down with streptavidin beads and prepared as general Illumina library. The Hi-C sample was sequenced paired-end 76 cycles on Illumina Hiseq 4000.

The reads were mapped to hg19 and processed using Juicer v1.5 ${ }^{42}$ with default parameters. Of $\sim 2.5$ billion Hi-C reads, $60.9 \%$ were Hi-C contact reads. Because many copies of DUX4 exist in the reference genome, in order to show the reasonable signals in the DUX4 region, no minimum mapping quality was required. The data matrices were further extracted from hic files with the dump function from juicer tools, and the data were visualized with the Hi-C viewer under development though the Pediatric Cancer (PeCan) data portal ${ }^{43}$.

The IGH intronic enhancer $(\mathrm{E} \mu)$ lies between the tandem repeat region and $I G H J$ region $^{44}$. In order to extract the contact reads between $\mathrm{E} \mu$ and Ig $\mu$ promoter from $\mathrm{Hi}-\mathrm{C}$ data, we defined $\mathrm{E} \mu$ and Ig $\mu$ promoter as the regions with highly enriched H3K27ac/ATAC-seq reads, marked as red boxes in Supplementary Fig. 8a and $8 \mathrm{~b}$. Only the read pairs that one read falls in $\mathrm{E} \mu$ and the other in the Ig $\mu$ promoter were used to evaluate the $\mathrm{E} \mu$-Ig $\mu$ promoter interaction. Because of multiple mapping issue for DUX4 region, to quantify E $\mu$-DUX4 interaction reads, all the read pairs that one read falls in $\mathrm{E} \mu$ and the other in any DUX4 array region annotated by Gencode V28lift37 were defined as the read pairs supported E $\mu$ DUX4 interaction.

H3K27ac HiChIP and data analysis. The Nalm6 cell line was cultured under recommended conditions to about $80 \%$ confluence. DNA from 10 million cells were crosslinked with $1 \%$ formaldehyde for $10 \mathrm{~min}$ at room temperature, then digested with 375 units of MboI, labeled by biotinylated nucleotides and proximity-ligated. After being sheared by Covaris, chromatin immunoprecipitated (ChIP) with H2K27ac antibody, the ChIP DNA was de-crosslinked and purified by Zymo Genomic GNA Clean \& Concentrator kit. Finally, after streptavidin beads pull-down and Illumina library construction by Kapa Hyper Prep Kit, hiChIP library was sequenced paired-end 76 cycles on Illumina NextSeq 500.

$\mathrm{HiC}-\mathrm{Pro}^{45}$ was used for $\mathrm{H} 3 \mathrm{~K} 27 \mathrm{ac} \mathrm{HiChIP}$ data analysis. Of 371.6 million reads, $75.6 \%$ were contact read. The same defined enhancer/promoter region and the same strategy for Hi-C data analysis were used to quantify $\mathrm{E} \mu-D U X 4$ interaction reads and $\mathrm{E} \mu$-Ig $\mu$ promoter interaction reads in $\mathrm{H} 3 \mathrm{~K} 27 \mathrm{ac}$ HiChIP data.

Capture-C and data analysis. Capture- $\mathrm{C}$ was designed to identify the regions interacting with $\mathrm{E} \mu$. There were three DpnII cutting sites (shown as green bars in Supplementary Fig. 9) in the enhancer region. Three oligos (Supplementary Table 1) were designed near the cutting sites to capture E $\mu$. Nalm6 was cultured to about $80 \%$ confluence and resuspended at a concentration of $10 \mathrm{e} 7$ cells in $10 \mathrm{ml}$ of medium. Cells were crosslinked with $2 \%$ formaldehyde and quenched by $0.125 \mathrm{M}$ glycine. After lysis, cells were kept on ice for $20 \mathrm{~min}$, digested with 500 units of DpnII, then underwent the proximity ligation. After reverse crosslinking, ligated DNA was purified, then sheared to $200 \mathrm{bp}$ and prepared as general indexed Illumina libraries. Two micrograms of different indexed samples were captured twice by specific biotinylated oligos, then PCR amplified streptavidin beads pulled down libraries. The NG capture-C libraries were sequenced on Miseq paired-end 151 cycles.

The data were analyzed using the pipeline capture- $\mathrm{C}^{46}$. In order to avoid very high read coverage in the oligo region during signal visualization, the reads at the extended $500 \mathrm{bp}$ region of the fragment (shown as a red bar in

Supplementary Fig. 9) were filtered. In addition to the interaction of $\mathrm{E} \mu$-Ig $\mu$ promoter and $\mathrm{E} \mu-D U X 4$, interactions were also detected between $\mathrm{E} \mu$ and IGHA/ IGHE (close to IGH 3' enhancer) (Fig. 3a). This result was consistent with current knowledge that $\mathrm{E} \mu$ is also in contact with the region near IGH $3^{\prime}$ enhancer ${ }^{47,48}$

The highest peak at DUX4 region and Ig $\mu$ promoter region were used to quantify E $\mu-D U X 4$ and the $\mathrm{E} \mu$-Ig $\mu$ interaction. Although many DUX4 copies exist in human genome, only one peak of 56 reads at DUX4L13 (the exact same sequence as the DUX4 sequence on our assembled IGH-DUX4 haplotype in Nalm6) was found (shown in Fig. 3a). The enrichment of Capture-C mapping to DUX4L13 can be explained by its $\geq 1$-bp difference with all other annotated DUX4 genes on hg19. To account for multiple mapping issue, we identified a total of 101 reads mapped to any DUX4 clusters based on the annotation of Gencode V28lift37 and mapped these 101 reads to the DUX4L13 nearby region (chr10:135,488,523-135,493,885 in hg19) using BWA ${ }^{34}$, resulting in an increase of DUX4L13 peak coverage to 75 . Therefore, the fold change of interaction intensity between the $\mathrm{E} \mu$-Ig $\mu$ promoter and $\mathrm{E} \mu-D U X 4$ was estimated to be 13 fold (953 vs. 75 ).
Genomic distance between E $\mu$ and Ig $\mu$ promoter/DUX4. From our assembled sequence of IGH-DUX4, the linear distance between E $\mu$ and DUX4 is $\sim 4 \mathrm{~kb}$ (Fig. 3b). Because the allele with Ig $\mu$ expression undergone VDJ recombination, most IGH V regions showed one allele deletion from Chromium WGS. However, a $\sim 126 \mathrm{~kb} V$ region was maintained in both alleles (Fig. 3a). Therefore, we estimated the linear distance between $\mathrm{E} \mu$ and Ig $\mu$ promoter is $\sim 140 \mathrm{~kb}$ (Fig. 3b).

A reciprocal translocation between chr10 subtelomere and IGH. A large-scale Hi-C plot between chr10 subtelomere $(1.4 \mathrm{Mb})$ and chr14 IGH $(1.4 \mathrm{Mb})$ was shown in Supplementary Fig. 5a. The translocation event "2 DUX4 copies were inserted into IGH D and J region" was shown as blue box. Most IGHV regions were deleted on the wild-type IGH allele based on well-known allelic exclusion of $I G H^{3}$. From the coverage of Chromium WGS, the big IGHV region " $\mathrm{C}$ " (marked as red bar) has one allele deletion, suggesting sequence at region " $\mathrm{C}$ " is on the non-wild-type IGH allele. The region "C" does not interact with IGHM/ enhancer region "A" (marked as green bar) but interact with chr10 subtelomere (marked as cyan bar) shown as yellow box, suggesting a reciprocal translocation between chr10 subtelomere and $I G H$ region. A model of the two translocation events between chr10 subtelomere and the non-wild-type (alternative) IGH allele was shown in Supplementary Fig. 5c.

Apoptosis assay. For the murine experiments, full-length DUX4 and ACTB cDNA were cloned into the MSCV-IRES-eGFP retroviral vector. Ecotropic envelope-pseudotyped virus was generated using the Phoenix ${ }^{\mathrm{xw}}$-Eco cell line (Thermo Fisher Scientific, Waltham, MA, USA) as per manufacturers instructions. Murine bone marrow from 4- to 6-week-old C57BL/6 mice was harvested, lineage depleted as per manufacturers instructions (Miltenyi Biotec, Bergisch Gladbach, Germany), and cultured in the presence of murine IL-3 (10 ng/ml), IL-6 $(30 \mathrm{ng} / \mathrm{ml})$, and SCF $(50 \mathrm{ng} / \mathrm{ml})$ (Pepro Tech US, Rocky Hill, NJ, US) for $24 \mathrm{~h}$ prior to transduction on RetroNectin (Takara Bio Inc.) ${ }^{49}$. Thirty-six to fortyeight hours following transduction with a single hit of virus, cells were harvested and analyzed by flow cytometry. Briefly, cells were washed and stained with annexin V conjugated to APC (BD Biosciences, San Jose, CA, USA) for $15 \mathrm{~min}$ at $4^{\circ} \mathrm{C}$, washed and resuspended in $4^{\prime}, 6$-diamidino-2-phenylindole containing solution ( $1 \mu \mathrm{g} / \mathrm{ml}$ DAPI) for analysis using FACS LSR II D (BD Biosciences, San Jose, CA, USA). All animal studies were maintained and treated in accordance with guidelines approved by the Institutional Animal Care and Use Committee (IACUC) at St. Jude Children's research hospital. For the Nalm6 cell line, a FLAG-tagged full-length DUX4 gene was cloned into the lentiviral construct pCDH (System Biosciences, Palo Alto, CA, USA). VSV-G pseudotyped virus was generated by transient transfection as per manufacturer's instructions (System Biosciences, Palo Alto, CA, USA). Thirty-six hours following transduction with a single hit of virus, cells were harvested and analyzed by flow cytometry and described above. GFP-positive populations were flow sorted for western blot analysis to confirm expression of DUX4 from the lentiviral construct. The details of gating strategy for mice bone marrow and Nalm6 can be found in Supplementary Fig. 10.

In IGH-DUX4 B-ALL patients, the C-terminal truncation of DUX4 caused by translocation varies significant, from 4aa to 200 aa (16aa for Nalm6) based on previous studies by us and others ${ }^{8-10}$ (https://pecan.stjude.cloud/ proteinpaint/DUX4). In a recent study, Dong et al. found that the wild-type DUX4 and IGH-DUX4 share similar DNA binding mechanism ${ }^{10}$. The crystal structure of Apo-DUX4HD2 and DNA-bound complex suggests that the Nterminal double homeobox domains are essential for IGH-DUX4 driven transactivation and B-ALL leukemogenesis. Combined, these data suggest the enhancer hijacking leading to expression of the DUX4 peptide is the real oncogenic effect. Therefore, the wild-type DUX4 was utilized in our apoptosis experiments.

Western blot analysis. Total protein was extracted from GFP positive flow sorted Nalm6 cells with RIPA lysis buffer and blotted with anti-FLAG (Sigma F3165; 10 $\mu \mathrm{g} / \mathrm{ml}$ ) and anti-Beta Actin (Abcam ab8227; 1:1000 dilution). Anti-mouse IgG superclonal-HRP (Invitrogen A28177; 1:5000 dilution) and anti-rabbit-HRP (Abcam ab6721-1; 1:3000 dilution) were used as secondary antibodies for FLAG and Beta Actin antibodies, respectively. Uncropped and unprocessed scans can be found in Supplementary Fig. 11.

Reporting summary. Further information on research design is available in the Nature Research Reporting Summary linked to this article.

\section{Data availability}

10X Chromium WGS, PacBio Iso-Seq data, and bisulfite sequencing data at IGHM region are available in NCBI BioProject PRJNA473990. H3K27ac ChIP-seq, Hi-C, HiChIP, Capture-C, and ATAC-seq data are available in the GEO database (GSE115494).

\section{Code availability}

The codes used in the analysis are available at https://github.com/liqingti/IGH-DUX4. 
Received: 29 June 2018 Accepted: 16 May 2019

Published online: 26 June 2019

\section{References}

1. Bergman, Y. \& Cedar, H. A stepwise epigenetic process controls immunoglobulin allelic exclusion. Nat. Rev. Immunol. 4, 753-761 (2004).

2. Perlot, T. \& Alt, F. W. Cis-regulatory elements and epigenetic changes control genomic rearrangements of the IgH locus. Adv. Immunol. 99, 1-32 (2008).

3. Vettermann, C. \& Schlissel, M. S. Allelic exclusion of immunoglobulin genes: models and mechanisms. Immunol. Rev. 237, 22-42 (2010).

4. Proudhon, C., Hao, B., Raviram, R., Chaumeil, J. \& Skok, J. A. Longrange regulation of $\mathrm{V}(\mathrm{D}) \mathrm{J}$ recombination. Adv. Immunol. 128, 123-182 (2015).

5. Russell, L. J. et al. IGH@ translocations are prevalent in teenagers and young adults with acute lymphoblastic leukemia and are associated with a poor outcome. J. Clin. Oncol. 32, 1453-1462 (2014).

6. Ott, G., Rosenwald, A. \& Campo, E. Understanding MYC-driven aggressive Bcell lymphomas: pathogenesis and classification. Hematol. Am. Soc. Hematol. Educ. Program 2013, 575-583 (2013).

7. Fonseca, R. et al. The recurrent IgH translocations are highly associated with nonhyperdiploid variant multiple myeloma. Blood 102, 2562-2567 (2003).

8. Yasuda, T. et al. Recurrent DUX4 fusions in B cell acute lymphoblastic leukemia of adolescents and young adults. Nat. Genet. 48, 569-574 (2016).

9. Zhang, J. et al. Deregulation of DUX4 and ERG in acute lymphoblastic leukemia. Nat. Genet. 48, 1481-1489 (2016).

10. Dong, X. et al. Structural basis of DUX4/IGH-driven transactivation. Leukemia 32, 1466-1476 (2018).

11. Zeng, W. et al. Specific loss of histone H3 lysine 9 trimethylation and HP1 $\gamma$ / cohesin binding at D4Z4 repeats is associated with facioscapulohumeral dystrophy (FSHD). PLoS Genet. 5, e1000559 (2009).

12. van Overveld, P. G. et al. Hypomethylation of D4Z4 in 4q-linked and non-4qlinked facioscapulohumeral muscular dystrophy. Nat. Genet. 35, 315-317 (2003).

13. Hendrickson, P. G. et al. Conserved roles of mouse DUX and human DUX4 in activating cleavage-stage genes and MERVL/HERVL retrotransposons. Nat. Genet. 49, 925-934 (2017).

14. van der Maarel, S. M., Tawil, R. \& Tapscott, S. J. Facioscapulohumeral muscular dystrophy and DUX4: breaking the silence. Trends Mol. Med. 17, 252-258 (2011).

15. Geng, L. N. et al. DUX4 activates germline genes, retroelements, and immune mediators: implications for facioscapulohumeral dystrophy. Dev. Cell 22, 38-51 (2012).

16. Peng, C. \& Eckhardt, L. A. Role of the Igh intronic enhancer Emu in clonal selection at the pre-B to immature B cell transition. J. Immunol. 191, 4399-4411 (2013).

17. Ma, X. et al. Pan-cancer genome and transcriptome analyses of 1,699 paediatric leukaemias and solid tumours. Nature 555, 371-376 (2018).

18. Levin-Klein, R. \& Bergman, Y. Epigenetic regulation of monoallelic rearrangement (allelic exclusion) of antigen receptor genes. Front Immunol. 5, 625 (2014).

19. Lajoie, B. R., Dekker, J. \& Kaplan, N. The Hitchhiker's guide to Hi-C analysis: practical guidelines. Methods 72, 65-75 (2015).

20. Gaillard, H., Garcia-Muse, T. \& Aguilera, A. Replication stress and cancer. Nat. Rev. Cancer 15, 276-289 (2015).

21. Serrano, M., Lin, A. W., McCurrach, M. E., Beach, D. \& Lowe, S. W. Oncogenic ras provokes premature cell senescence associated with accumulation of p53 and p16INK4a. Cell 88, 593-602 (1997).

22. Kowaljow, V. et al. The DUX4 gene at the FSHD1A locus encodes a proapoptotic protein. Neuromuscul. Disord. 17, 611-623 (2007).

23. Walker, B. A. et al. Characterization of IGH locus breakpoints in multiple myeloma indicates a subset of translocations appear to occur in pregerminal center B cells. Blood 121, 3413-3419 (2013).

24. Trageser, D. et al. Pre-B cell receptor-mediated cell cycle arrest in Philadelphia chromosome-positive acute lymphoblastic leukemia requires IKAROS function. J. Exp. Med. 206, 1739-1753 (2009).

25. Geng, H. et al. Self-enforcing feedback activation between BCL6 and pre-B cell receptor signaling defines a distinct subtype of acute lymphoblastic leukemia. Cancer Cell 27, 409-425 (2015).

26. Amin, A. D., Rajan, S. S., Groysman, M. J., Pongtornpipat, P. \& Schatz, J. H. Oncogene overdose: too much of a bad thing for oncogene-addicted cancer cells. Biomark. Cancer 7, 25-32 (2015).

27. Cruz, J. M. et al. Protein kinase A activation inhibits DUX4 gene expression in myotubes from patients with facioscapulohumeral muscular dystrophy. J. Biol. Chem. 293, 11837-11849 (2018).
28. Roberts, K. G. et al. Targetable kinase-activating lesions in Ph-like acute lymphoblastic leukemia. N. Engl. J. Med. 371, 1005-1015 (2014).

29. Dobin, A. et al. STAR: ultrafast universal RNA-seq aligner. Bioinformatics 29, 15-21 (2013).

30. Anders, S., Pyl, P. T. \& Huber, W. HTSeq-a Python framework to work with high-throughput sequencing data. Bioinformatics 31, 166-169 (2015).

31. Weisenfeld, N. I., Kumar, V., Shah, P., Church, D. M. \& Jaffe, D. B. Direct determination of diploid genome sequences. Genome Res. 27, 757-767 (2017).

32. Kent, W. J. BLAT-the BLAST-like alignment tool. Genome Res. 12, 656-664 (2002).

33. Buenrostro, J. D., Wu, B., Chang, H. Y. \& Greenleaf, W. J. ATAC-seq: A method for assaying chromatin accessibility genome-wide. Curr. Protoc. Mol. Biol. 109, 2129 21-21 2929 (2015).

34. Li, H. \& Durbin, R. Fast and accurate short read alignment with BurrowsWheeler transform. Bioinformatics 25, 1754-1760 (2009).

35. McKenna, A. et al. The Genome Analysis Toolkit: a MapReduce framework for analyzing next-generation DNA sequencing data. Genome Res. 20, 1297-1303 (2010).

36. Li, H. et al. The sequence alignment/Map format and SAMtools. Bioinformatics 25, 2078-2079 (2009).

37. Buenrostro, J. D., Giresi, P. G., Zaba, L. C., Chang, H. Y. \& Greenleaf, W. J. Transposition of native chromatin for fast and sensitive epigenomic profiling of open chromatin, DNA-binding proteins and nucleosome position. Nat. Methods 10, 1213-1218 (2013).

38. Robinson, J. T. et al. Integrative genomics viewer. Nat. Biotechnol. 29, 24-26 (2011).

39. Landt, S. G. et al. ChIP-seq guidelines and practices of the ENCODE and modENCODE consortia. Genome Res. 22, 1813-1831 (2012).

40. Kharchenko, P. V., Tolstorukov, M. Y. \& Park, P. J. Design and analysis of ChIP-seq experiments for DNA-binding proteins. Nat. Biotechnol. 26, 1351-1359 (2008).

41. Xi, Y. \& Li, W. BSMAP: whole genome bisulfite sequence MAPping program. BMC Bioinforma. 10, 232 (2009).

42. Durand, N. C. et al. Juicer provides a one-click system for analyzing loopresolution Hi-C experiments. Cell Syst. 3, 95-98 (2016).

43. Zhou, X. et al. Exploring genomic alteration in pediatric cancer using ProteinPaint. Nat. Genet. 48, 4-6 (2016).

44. Georgiou, G. et al. The promise and challenge of high-throughput sequencing of the antibody repertoire. Nat. Biotechnol. 32, 158-168 (2014).

45. Servant, N. et al. HiC-Pro: an optimized and flexible pipeline for $\mathrm{Hi}-\mathrm{C}$ data processing. Genome Biol. 16, 259 (2015).

46. Davies, J. O. et al. Multiplexed analysis of chromosome conformation at vastly improved sensitivity. Nat. Methods 13, 74-80 (2016).

47. Roy, A. L., Sen, R. \& Roeder, R. G. Enhancer-promoter communication and transcriptional regulation of Igh. Trends Immunol. 32, 532-539 (2011).

48. Garot, A. et al. Sequential activation and distinct functions for distal and proximal modules within the IgH 3 ' regulatory region. Proc. Natl Acad. Sci. USA 113, 1618-1623 (2016)

49. Dang, J. et al. AMKL chimeric transcription factors are potent inducers of leukemia. Leukemia 31, 2228-2234 (2017).

\section{Acknowledgements}

We thank Shuoguo Wang and Ti-Cheng Chang for help with 10X Chromium WGS analysis, and Drs. Hongbo Chi and Yongqiang Feng for critical review of the manuscript. We thank Lily Maxham and Janet Partridge for confirming DUX4 toxicity experiment using inducible expression system in Nalm6. This research was supported by the National Cancer Institute of the National Institutes of Health under Award Number R01CA216391. The content is solely the responsibility of the authors and does not necessarily represent the official views of the National Institutes of Health. This research was also supported by Cancer Center Support Grant P30CA021765 from the National Institutes of Health and in part by the American Lebanese Syrian Associated Charities (ALSAC).

\section{Author contributions}

J.Z. conceived the project. J.Z. and L.T. designed the study. L.T., B.X., X.M., Y.L., P.S., and X.Z. analyzed the data. S.Newman provided data analysis advice. Y.S., B.J., and J.E designed and performed 10X Chromium WGS/ATAC-seq/Hi-C/HiChIP/Capture-C experiment. E.T, T.H., and M.A. performed Iso-Seq data. T.A.G. designed the apoptosis experiments which were performed by S.Nance and J.D. L.D. helped on western blot experiment. C.L. advised and gave support to the experiment. All authors contributed to the writing, editing, and completion of the manuscript. 


\section{Additional information}

Supplementary Information accompanies this paper at https://doi.org/10.1038/s41467019-10637-8

Competing interests: E.T., T.H., and M.A. are employed by Pacific Biosciences. The other authors declare no completing interests.

Reprints and permission information is available online at http://npg.nature.com/ reprintsandpermissions/

Peer review information: Nature Communications thanks Florence Nguyen-Khac and other anonymous reviewer(s) for their contribution to the peer review of this work. Peer reviewer reports are available.

Publisher's note: Springer Nature remains neutral with regard to jurisdictional claims in published maps and institutional affiliations. (c) Open Access This article is licensed under a Creative Commons Attribution 4.0 International License, which permits use, sharing, adaptation, distribution and reproduction in any medium or format, as long as you give appropriate credit to the original author(s) and the source, provide a link to the Creative Commons license, and indicate if changes were made. The images or other third party material in this article are included in the article's Creative Commons license, unless indicated otherwise in a credit line to the material. If material is not included in the article's Creative Commons license and your intended use is not permitted by statutory regulation or exceeds the permitted use, you will need to obtain permission directly from the copyright holder. To view a copy of this license, visit http://creativecommons.org/ licenses/by/4.0/.

(C) The Author(s) 2019 